\title{
Article
}

\section{Frequency Selective Buildings Through Frequency Selective Surfaces}

Raspopoulos, Marios and Stavrou, Stavros

Available at http://clok.uclan.ac.uk/17909/

Raspopoulos, Marios ORCID: 0000-0003-1513-6018 and Stavrou, Stavros (2011) Frequency Selective Buildings Through Frequency Selective Surfaces. IEEE Transactions on Antennas and Propagation, 59 (8). pp. 2998-3005. ISSN 0018-926X

It is advisable to refer to the publisher's version if you intend to cite from the work. http://dx.doi.org/10.1109/TAP.2011.2158779

For more information about UCLan's research in this area go to

http://www.uclan.ac.uk/researchgroups/ and search for <name of research Group>.

For information about Research generally at UCLan please go to http://www.uclan.ac.uk/research/

All outputs in CLoK are protected by Intellectual Property Rights law, including Copyright law. Copyright, IPR and Moral Rights for the works on this site are retained by the individual authors and/or other copyright owners. Terms and conditions for use of this material are defined in the policies page. 


\title{
Frequency Selective Buildings through Frequency Selective Surfaces
}

\author{
Marios Raspopoulos, Stavros Stavrou, Member, IEEE
}

\begin{abstract}
This work proposes the deployment of Frequency Selective Surfaces (FSS) in indoor wireless environments and investigates their effect on radio wave propagation. FSS can be deployed to selectively confine radio propagation in indoor areas, by artificially increasing the radio transmission loss naturally caused by building walls. FSS can also be used to channel radio signals into other areas of interest. Simulations and measurements have been carried out in order to verify the frequency selectivity of the FSS. Practical considerations regarding the deployment of FSS on building walls and the separation distance between the FSS and the supporting wall have been also investigated. Finally, a controlled, small-scale indoor environment has been constructed and measured in an anechoic chamber in order to practically verify this approach through the usage of ray tracing techniques.
\end{abstract}

Index Terms-Frequency Selective Surfaces, Buildings, Indoor Radio Communication, Radio Propagation, Repeaters.

\section{INTRODUCTION}

$\mathbf{R}$ ADIO signals generally propagate through reflections, refractions, diffractions and scattering. Transmitted signal components arrive at the point of interest with various amplitudes and phases and combine together to produce the received signal. Due to these multipath arriving components, the instantaneous received signal strength can typically vary as much as $20-30 \mathrm{~dB}$ over a fraction of a wavelength due to constructive and destructive contributions [1]. In a typical indoor environment the received signal is mainly attenuated due to reflections and transmissions through building materials. Building penetration loss depends on various variables associated with the building architecture, including but not limited to the building structure, the building walls periodicity and their electrical properties, the angle of incidence etc [2], [3], [4], [5].

Depending on the application, there are cases where the radio signals have to be confined in designated areas of interest, improving in this way wireless security. Such signal confinement can also assist interference management. However, there are also cases where these signals have to be amplified in order to increase wireless coverage and system reliability.

The literature proposes various methods to increase coverage. These include leaky feeders, active and passive repeaters, distributed antennas, etc. Leaky feeders principle of operation is based on the leakage fields arising from specially made coaxial cables, which are usually deployed in large pathways

M. Raspopoulos is with Sigint Solutions Ltd, Nicosia, Cyprus (email:m.raspopoulos@sigintsolutions.com).

S. Stavrou is with Open University Cyprus.

Manuscript received October 2, 2010 (e.g. tunnels) [6], [7]. Despite that they have been mainly used in tunnels and mines [8] they have also found applications in indoor environments, especially in offices with lengthy corridors [9], [10]. The active repeaters are based on the idea of receiving the signal in a particular location, amplifying and re-transmitting it to the same or another direction. However, this technique requires power and good isolation between the receiving and re-transmitting antennas [11], otherwise the system might become oscillatory. Also, with active repeaters, the received noise and interference is re-radiated on both the forward and the reverse link.

The technique investigated in this paper is to utilise Frequency Selective Surfaces (FSS) as isolators or even as passive repeaters, in indoor environments [12]. The passive repeater concept is based on the assumption that the mean signal strength received due to a reflected contribution from a FSS would be higher from the one received from any other object/material that does not produce a strong reflection [13], [14], [15], [16]. Alternatively, FSS can be used to provide radio isolation by selectively rejecting a frequency range, thus reducing interference between adjacent co-channel wireless systems. Wireless security can be also improved by minimizing the spillover of radio waves outside designated areas. Similar behavior can be achieved, if instead of FSS, a metallic surface is used as the reflecting interface. However, the drawback of such a method is that it will unselectively block and/or reflect the frequency range of multiple wireless systems [17]. FSS can be deployed in such a way so as WLAN signals can be confined in an indoor environment while GSM signals can be allowed to enter and exit the building with a zero or minimal effect. The authors of this paper have made a first attempt to demonstrate the principle of the application of FSS in wireless environments to improve and/or restrict coverage in specific areas in 2005 and 2006 [12], [18]. In 2006, Sung has demonstrated through in-situ measurement results over a frequency range of $2.3-7 \mathrm{GHz}$ that a frequency selective wall can be successfully created for UNII (Unlicensed National Information Infrastructure) [19], [20]. The experiments have been carried out in two adjacent rooms, separated by a wall which is transformed into frequency selective by attaching a band-stop FSS. Deployment practicalities such as misalignment and overlapping are investigated. In this paper, we investigate the application of FSS through the use of Ray Tracing simulations which incorporate the behavior of FSS. This gives the flexibility to predict the behavior of a Frequency Selective Building where FSS are deployed in more than one wall. Practical considerations regarding the deployment of FSS on building walls and the separation 
distance between the FSS and the supporting wall have been also investigated using CFDTD simulations. Section II of this paper presents some of the FSS behavior issues related to this work. These include the performance of various element shapes, the effect of angle of incidence and typical building materials on the transmission and reflection characteristics of FSS, when these are placed at various distances behind the FSS. Typical modeled and measured responses are also provided. Section III presents results from a custom-written Ray Tracing engine, developed and utilized for the purpose of this investigation. Section IV presents a small scale FSS indoor environment that has been constructed and tested in an anechoic chamber. This experiment practically verifies the usefulness of this investigation and the usage of ray tracing for predicting radio propagation in FSS environments. Section V presents a more detailed investigation of radio propagation in a typical FSS indoor environment through the use of the ray tracing engine, by incorporating the FSS interface behavior.

\section{Frequency Selective Surfaces}

FSS are planar periodic structures which consist of thin conducting elements, usually printed on a dielectric substrate. They behave as passive electromagnetic filters, selectively reflecting a desired frequency band. One of the most important factors influencing the FSS response is the element shape [21], [22], [23]. Their response can also be affected by the element size, the permittivity and thickness of the substrate, the angle of incidence and the gap between the elements. Various approaches have been developed for analyzing the FSS behavior. Examples include the Equivalent Circuit Method (ECM) [27], [28], [29], the Method of Moments (MoM), the Finite Difference Time Domain method (FDTD) and the Conformal Finite Difference Time Domain method (CFDTD) [24], [25], [26].

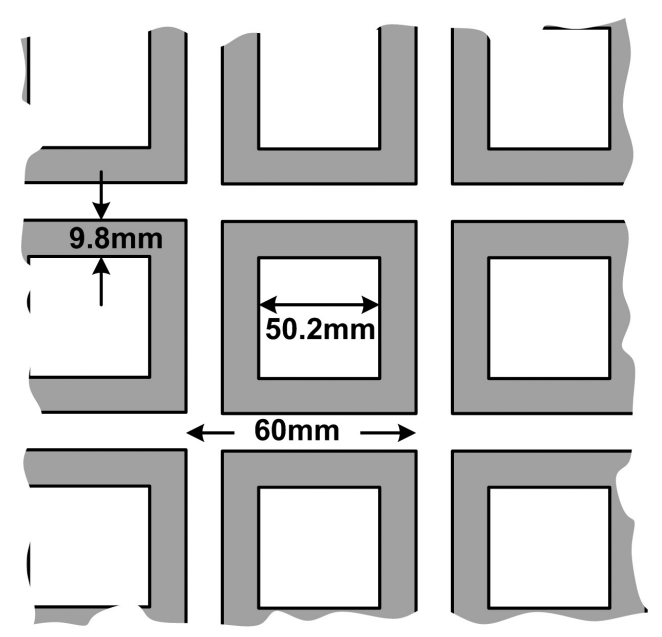

Fig. 1. Square-Loop FSS dimensions (substrate thickness: 1.6mm)

For the purpose of this investigation a square-loop FSS was designed and fabricated on FR4 dielectric $\left(\varepsilon_{r}=4.55, \tan \delta=0.0175\right)$. In this work the square loop design was selected mainly because of its superiority with regards to its angular insensitivity over the other element shapes. Table I presented by $\mathrm{Wu}$ in [31] presents a typical comparison between different elements. Fig. 1 shows the dimensions of the fabricated square-loop FSS used.

TABLE I

PERFORMANCE OF VARIOUS FSS ELEMENT SHAPES

\begin{tabular}{lcccc}
\hline \hline $\begin{array}{l}\text { Element } \\
\text { Type }\end{array}$ & $\begin{array}{c}\text { Angular } \\
\text { Insensitivity }\end{array}$ & $\begin{array}{c}\text { Cross- } \\
\text { Polarization }\end{array}$ & $\begin{array}{c}\text { Larger } \\
\text { Bandwidth }\end{array}$ & $\begin{array}{c}\text { Small Band } \\
\text { Separation }\end{array}$ \\
\hline $\begin{array}{l}\text { Loaded } \\
\text { Dipole }\end{array}$ & 1 & 2 & 1 & 1 \\
\hline $\begin{array}{l}\text { Jerusalem } \\
\text { Cross }\end{array}$ & 2 & 3 & 2 & 2 \\
\hline Rings & 1 & 2 & 1 & 1 \\
\hline Tripole & 3 & 3 & 3 & 2 \\
\hline $\begin{array}{l}\text { Cross } \\
\text { Dipole }\end{array}$ & 3 & 3 & 3 & 3 \\
\hline $\begin{array}{l}\text { Square } \\
\text { Loop }\end{array}$ & 1 & 1 & 1 & 1 \\
\hline Dipole & 4 & 1 & 4 & 1 \\
\hline
\end{tabular}

Ratings: $1=$ best, $2=2$ nd best, etc.

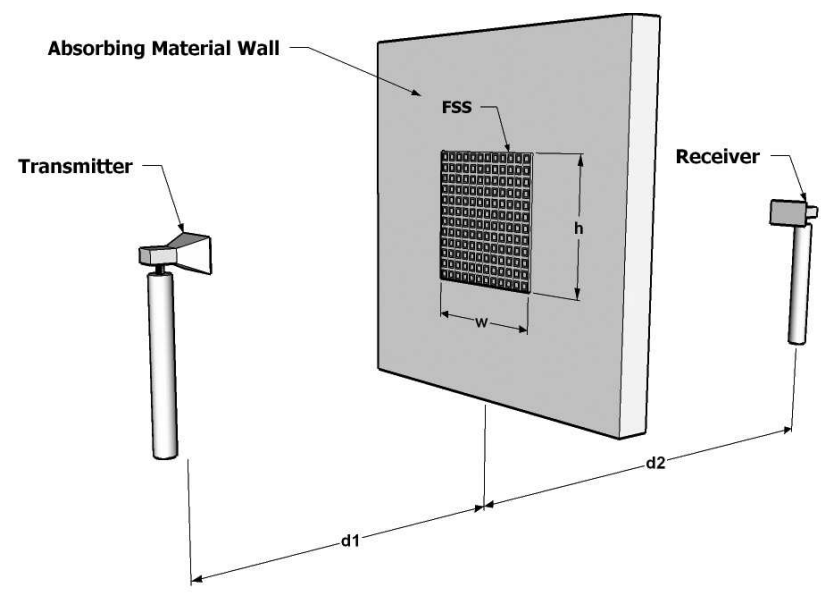

Fig. 2. Anechoic Chamber setup for FSS transmission loss measurements

To characterize the FSS response, measurements were carried out in the anechoic chamber of the Centre for Communication Systems Research in the University of Surrey. Double Ridge Guide linearly polarized Horn Antennas have been used for transmission and reception and a Vector Network Analyzer (Rohde and Schwarz VNA ZVCE $20 \mathrm{KHz}-8 \mathrm{GHz}$ ) was used to sweep the frequency between 1-4 GHz. These directional antennas have frequency independent characteristics in this frequency range with relatively constant gain at $9 \mathrm{dBi}$ and 40 degrees beamwidth. The measurement setup shown in Fig. 2 consists of a wooden board, covered with aluminium foil and absorbing material, which has a $72 \mathrm{~cm} \times 72 \mathrm{~cm}$ aperture in the middle for FSS placement. Given that the horn antennas' maximum dimension is $D=0.22 \mathrm{~m}, d_{1}$ have and $d_{2}$ in Fig. 2 have been selected to be $1.3 \mathrm{~m}$ in order to ensure that the FSS lies in the far-field region of the two antennas based on $d_{\text {farfield }} \geq 2 D^{2} / \lambda$, where $\lambda$ is the wavelength $(12.5 \mathrm{~cm})$. 
In order to ensure that all the contributions arriving at the receiving antenna are all in phase, the $60 \%$ of the 1st Fresnel Zone should be kept unobstructed. This is done by ensuring that $h / 2 \geq r_{0.6}$ and $w / 2 \geq r_{0.6}$ where $r_{n}$ is the radius of the $n$th Fresnel Zone given by $r_{n}=\sqrt{n \lambda d_{1} d_{2} /\left(d_{1}+d_{2}\right)}$. For this setup $r_{0.6}=0.34 \mathrm{~m}$ which satisfies the aforementioned conditions. To eliminate the effects of any possible edge diffraction around the edges of the aperture that the FSS is placed and the edges of the absorbing wall, these have been covered with absorbing material. To further eliminate these diffraction effects, the results have been time-gated using a 5ns timing window.

The simulations were performed using the CFDTD solver presented in [25] and [26]. For simulation purposes, the FR4 effective dielectric constant was set to 2.775 as suggested by $\varepsilon_{\text {eff }}=\left(1+\varepsilon_{r}\right) / 2$ [17], [25]. The assumption in these CFDTD simulations is that the current distribution of the truncated FSS $(72 \mathrm{~cm} \times 72 \mathrm{~cm})$ is the same as the one of an infinite structure and zero outside the finite structure [30]. This means that the infinite simulation does not consider the edge effects which have been eliminated in the chamber by using absorbing material and time gating.

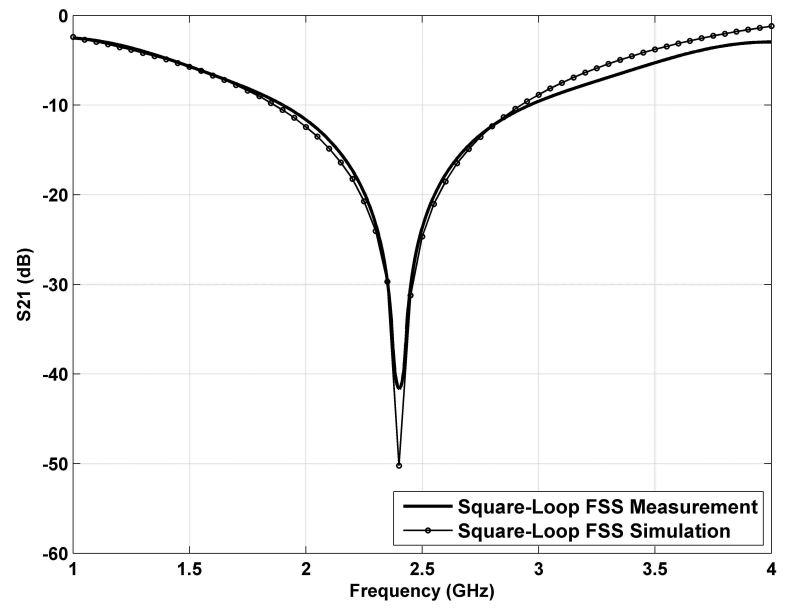

Fig. 3. Transmission through Square-Loop FSS at normal incidence

Figure 3 presents the simulated and measured transmission characteristics of the square-loop FSS under normal angle of incidence. The measurement result is in close agreement with the simulated result which gives us a degree of confidence on the CFDTD method used in addition to the published results of [25].

Figures 4 and 5 present CFDTD [25], [26] simulation results of the transmission $S_{21}$ and reflection $S_{11}$ characteristics of the square-loop FSS, under four angles of incidence $\left(0^{\circ}, 18^{\circ}, 36^{\circ}\right.$ and $\left.54^{\circ}\right)$, for TE and TM polarisations respectively. It is clear that as the angle of incidence is varied, the transmission and reflection characteristics will vary as well. Thus, when designing a FSS for a particular scenario, this angular sensitivity has to be taken into account in order to correctly model the radio wave interaction with the FSS deployed in the modelled environment.
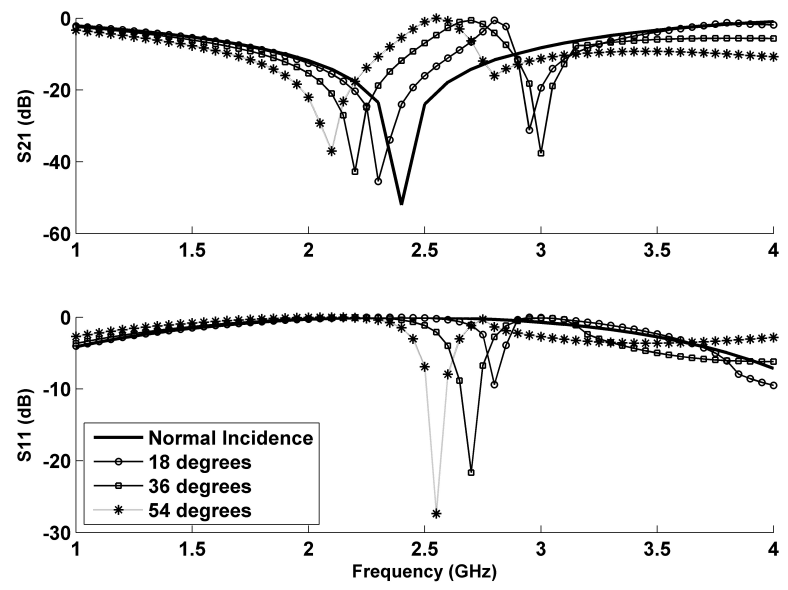

Fig. 4. TE Transmission $\left(S_{21}\right)$ and TE Reflection $\left(S_{11}\right)$ for square loop FSS under various angles of incidence.
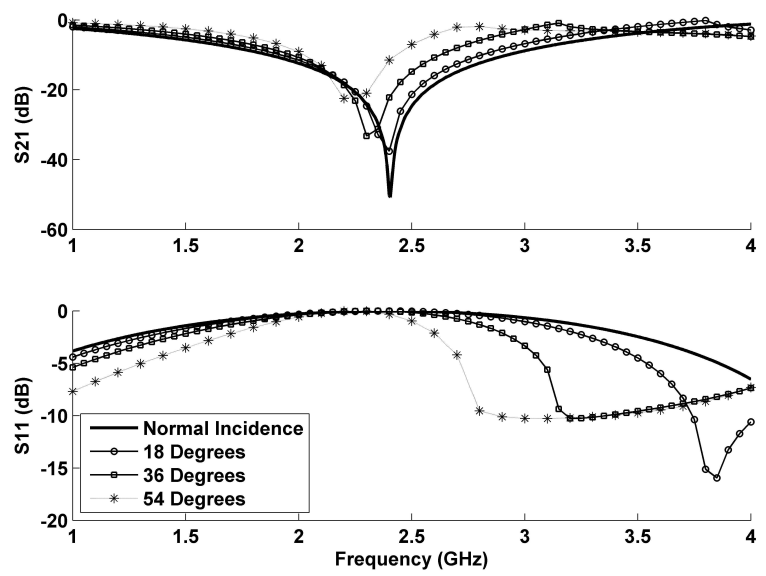

Fig. 5. TM Transmission $\left(S_{21}\right)$ and TE Reflection $\left(S_{11}\right)$ for square loop FSS under various angles of incidence.

For the purpose of this investigation the FSS was deployed on the building interfaces. Literature reports that when a FSS is placed on a dielectric medium, its standalone frequency response will change [17], [32]. Therefore, the interaction of building walls and FSS has to be investigated when considering this kind of application.

Figure 6 shows the building materials effect on the frequency response of the FSS. The results indicate that there is a decrease on the tuning frequency of the FSS when the latter is attached to the building material. This is due to the fact that Floquet modes which decay exponentially with distance from the elements, still have significant amplitudes at the boundary, hence modifying their relative amplitudes and the energy stored close to the array and thus affecting the resonant frequency [32] - [35]. To further investigate this effect, simulations and measurements have been performed by varying the distance between the FSS and the material. Figure 7 presents simulations and measurement results after varying the air gap between the FSS and a wooden building wall (MDF with $\varepsilon_{r}=1.9$ and $\sigma=0.008$ ). 


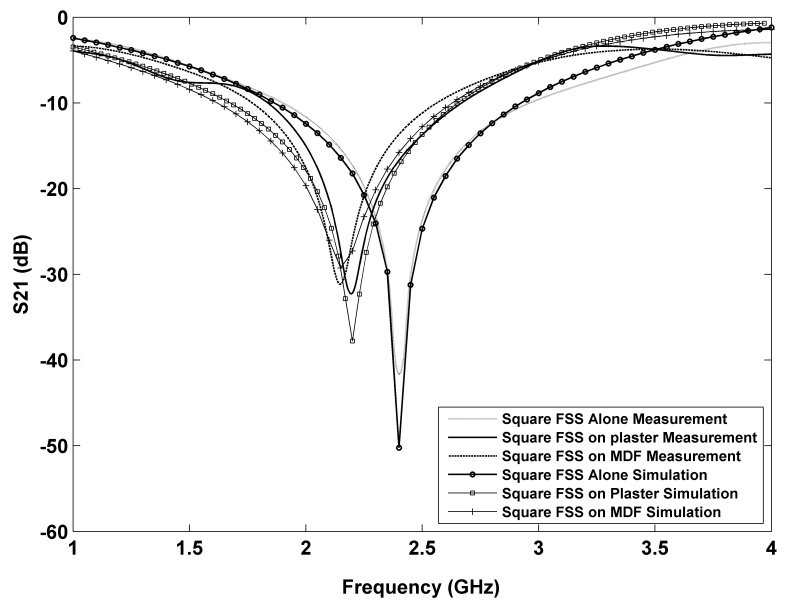

Fig. 6. Effect of wood and plaster when these are attached on the square-loop FSS

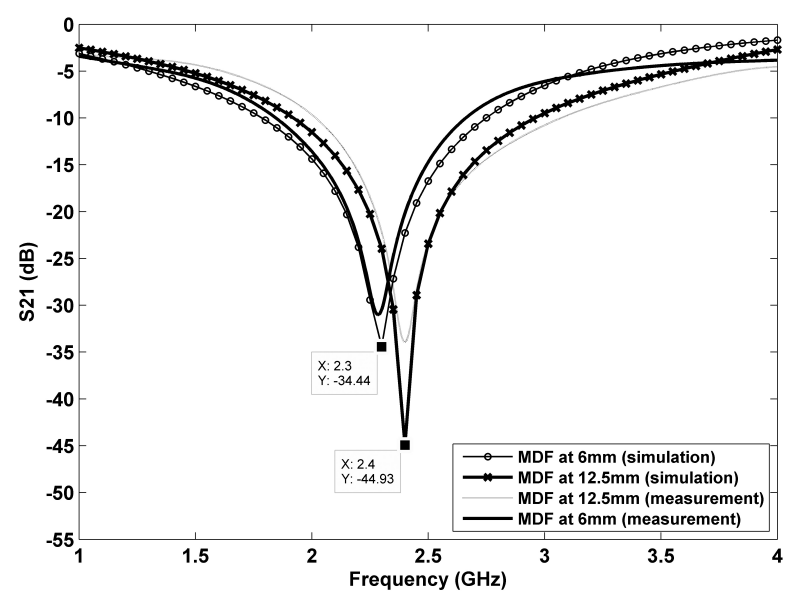

Fig. 7. Effect of varying the distance between the square-loop FSS and a wooden board

Figure 8 indicates that when the air gap is bigger than $\lambda / 10$ the effect of the wooden wall on the tuning frequency of the FSS is eliminated [34].

\section{RAY TRACING MODEL}

In order to study the effect of deploying FSS in an indoor environment, a 3D Ray Tracing model was developed to simulate a simple scenario with and without the FSS. The application of Ray Tracing falls into the category of deterministic or site-specific modeling [36], [37], which is very well suited for this work. Ray Tracing is based on Geometrical Optics (GO) and is used to identify all the possible ray paths between the transmitter and the receiver. Typical Ray Tracing algorithms include the Shooting and Bouncing Ray (SBR) and the Image method.

For the purpose of this work, a Ray Tracing algorithm was implemented in MATLAB, based on the image method. For this method, for a given source point and a facet, the reflected rays on the facet can be considered as being directly radiated from a virtual source called the image source, which

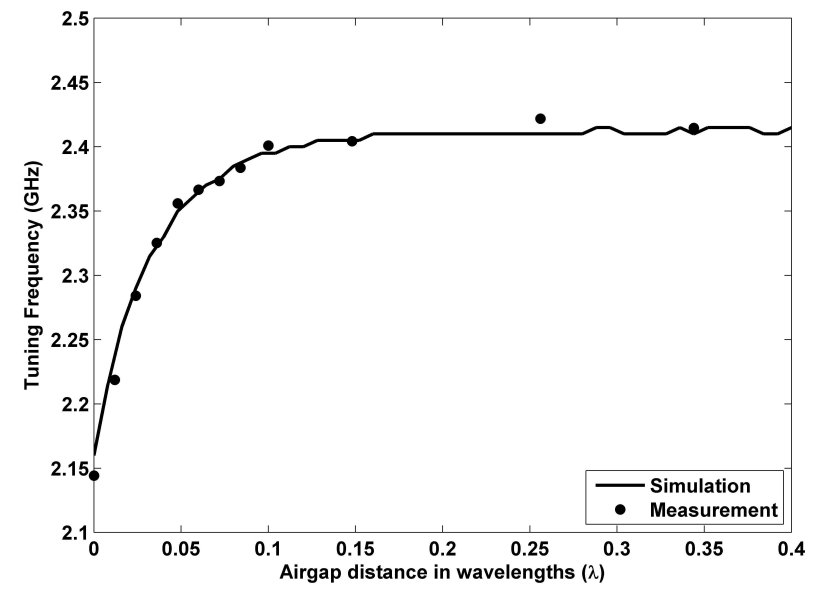

Fig. 8. Fig. 8. Simulation and Measurement of the effect of the air gap between the square-loop FSS and a 9mm-thick wooden (MDF) board on the tuning frequency

is symmetrical to the real source with respect to the facet. The first step during the implementation of the algorithm was to unambiguously define the environment under investigation in terms of its geometrical and morphological characteristics. These two descriptions are integrated into the faceted model, where every building interface is represented by a polygonshaped facet, which geometrically and morphologically describes the interface.

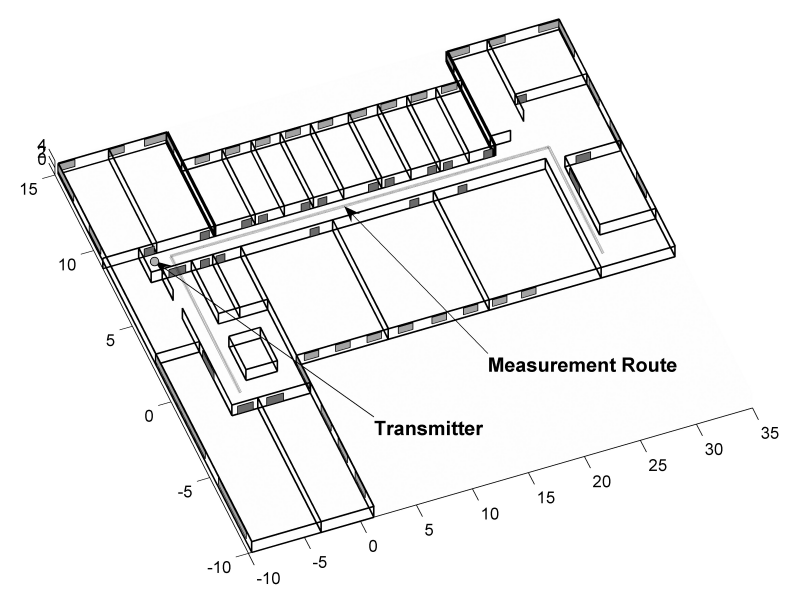

Fig. 9. Measurement route for validation of the Ray Tracing Tool

In order to validate the basic Ray Tracing algorithm prior to the investigation of the effect of FSS, a real scenario (second floor of the Centre for Communication Systems Research, CCSR, University of Surrey) was simulated and radio measurements were performed along the measurement route depicted in Figure 9. The basic Ray Tracing modeling approach was also compared with results obtained from a commercial Ray Tracing simulator by RemCom (Wireless Insite), which utilizes the SBR method. Both Ray Tracing simulation tools consider up to six reflections, all the pos- 
sible refractions and one UTD diffraction [38]. The electrical parameters of the scenario interfaces, used in both simulating tools, are tabulated in Table II. Typical constitutive parameters for different materials can be found in [3].

TABLE II

ELECTRICAL PARAMETERS USED FOR CCSR INTERFACE

\begin{tabular}{lcccc}
\hline \hline $\begin{array}{l}\text { Interface } \\
\text { Type }\end{array}$ & Material & $\begin{array}{c}\text { Relative } \\
\text { Electrical } \\
\text { Permittivity }\end{array}$ & $\begin{array}{c}\text { Conductivity } \\
\sigma\end{array}$ & $\begin{array}{c}\text { Thickness } \\
\text { (meters) }\end{array}$ \\
\hline $\begin{array}{l}\text { External } \\
\text { Wall }\end{array}$ & Brick & 5.5 & 0.018 & 0.30 \\
\hline $\begin{array}{l}\text { Floor and } \\
\text { Ceiling }\end{array}$ & Concrete & 8 & 0.01 & 0.40 \\
\hline $\begin{array}{l}\text { Internal } \\
\text { Wall }\end{array}$ & Plaster & 3.5 & 0.015 & 0.15 \\
\hline Door & Wood & 2 & 0.008 & 0.10 \\
\hline
\end{tabular}

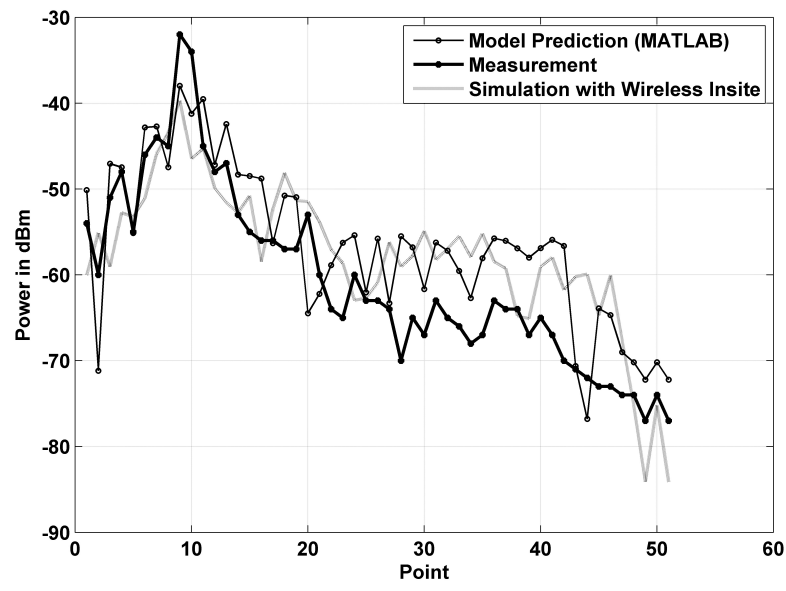

Fig. 10. Comparison between measurements and modeling

Figure 10 shows the measurement route consisting of 51 (both LOS and NLOS) averaged measurement points. Measurements were carried out with a portable spectrum analyzer from Rohde and Schwarz (FSP 30). The transmitter used was a Rohde Schwarz signal generator transmitting a $10 \mathrm{dBm}$ CW signal at a frequency of $2.4 \mathrm{GHz}$. Dipole antennas were used for transmitting and receiving. Figure 10 shows the comparison of the theoretical predictions and the measurement set. The recorded set of measurements has indicated a mean square error of around $4 \mathrm{~dB}$ and a model error standard deviation of around $5 \mathrm{~dB}$. One reason for the minor differences between the theoretical predictions and the measurements is the possible inaccurate use of the constitutive parameters and the presence of indoor clutter [2], [3].

\section{FSS - RAY TRACING IN ANEChOIC ENVIRONMENT}

In order to verify the applicability of Ray Tracing for predicting radio propagation in FSS indoor environments, an experiment was carried out in an anechoic chamber as depicted in Figures 11 and 12. The transmitting antenna was placed outside the chamber so as a LOS component could not be received by the identical receiving antenna $(1-8 \mathrm{GHz}$ horn antennas with 40 degrees beamwidth), which was placed inside the chamber. Two wooden boards were placed inside the chamber to direct the ray components to the receiving antenna (Figures 11 and 12). With this setup, the rays launched by the transmitter reflect on board 1 and 2 and then reach the receiver. The average distance traveled by the rays after the two reflections is 6.3 meters, corresponding to a path loss of $56 \mathrm{~dB}$ at $2.4 \mathrm{GHz}$ and the angle of incidence varies between 45 to 60 degrees.

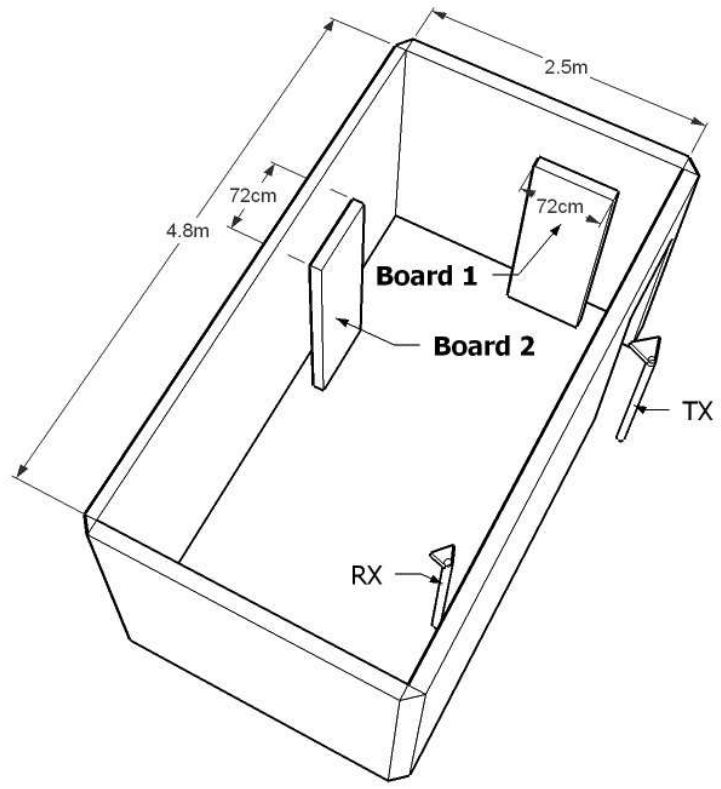

Fig. 11. Anechoic Chamber experiment setup

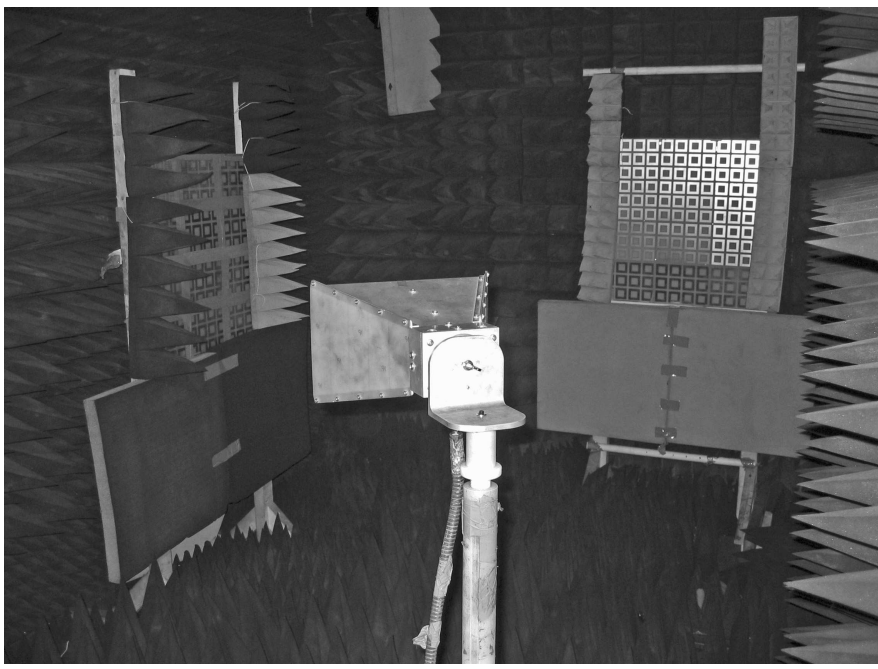

Fig. 12. Anechoic Chamber experiment setup

Three cases have been measured; reflectors (1) are made of wood, (2) are covered with metallic surfaces and (3) are covered with FSS as depicted in Figure 11. Figure 13 presents the results of these measurements. These results have been time-gated to isolate only the reflected components and discard any other possible contributions. It is clear that there is a 
significant increase of the field strength at $2.4 \mathrm{GHz}$ for the FSS case compared to the results obtained from the wooden board case. The received power at $2.4 \mathrm{GHz}$ is the same as the one for the metallic case, but significantly lower for any other frequencies. This result verifies the application of the Frequency Selective Surface as a passive repeater, used to increase signal coverage for specific frequencies of operation without affecting the operation of other systems operating on other frequencies. Also this experiment and the comparison with the theoretical Ray Tracing results demonstrates that Ray Tracing can be applied to predict radio propagation in FSS environments. The inclusion of FSS makes the problem not entirely ray-optical and therefore the developed Ray Tracing model was modified to incorporate the theoretical behaviour of FSS by replacing the Fresnel reflection and transmission coefficient calculations with pre-calculated CFDTD angledependent FSS coefficients. Diffraction effects in this case have been neglected and that is why the edges of the boards in the chamber have been covered with absorbing material.

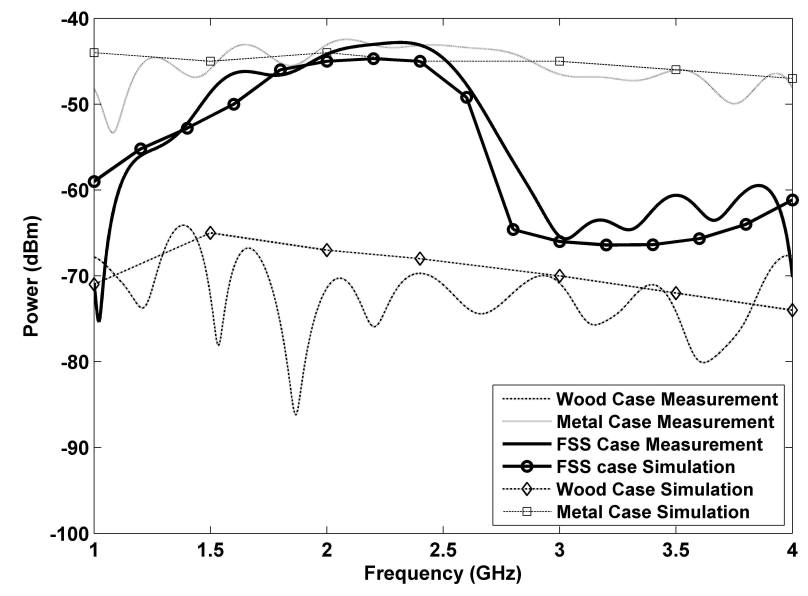

Fig. 13. Anechoic Chamber results for the 3 cases under investigation

\section{IMPACT OF FSS IN AN INDOOR ENVIRONMENT}

After the Ray Tracing algorithms were verified, a simpler indoor scenario was developed (Figure 14) in order to study the theoretical behavior of the FSS when these are incorporated on the building interfaces. The facets in the simulated environment were also described in such a way as to take into account the FSS behavior, under various angles of incidence, as suggested by the CFDTD method. The square-loop FSS reflection and transmission coefficients for all possible angles of incidence were calculated and incorporated into the Ray Tracing model. The Ray Tracing model considers up to six reflections, all the possible refractions and one UTD diffraction around the wall edges. It is assumed that the FSS are not placed around the edges of building walls and therefore the diffraction effects are calculated using the UTD solution of [38]. As already mentioned in Section II, when the air gap between the FSS and the building material is bigger than $\lambda / 10$, then there is no significant effect on the FSS response [34]. For this reason the square-loop FSS was placed $12.5 \mathrm{~mm}$ away from all the building materials (i.e. brick, concrete and plaster board). An illustrative example is shown in Figure 15 where the square loop FSS is placed $12.5 \mathrm{~mm}$ away from plaster board.

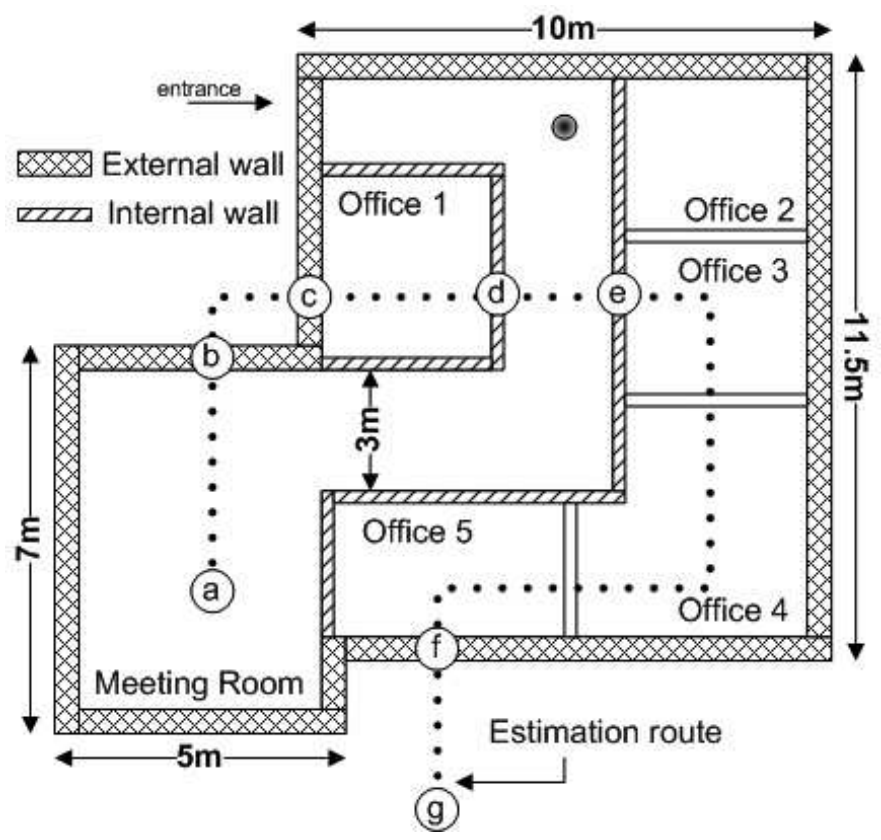

Fig. 14. Scenario under investigation $($ Height $=3 \mathrm{~m})$

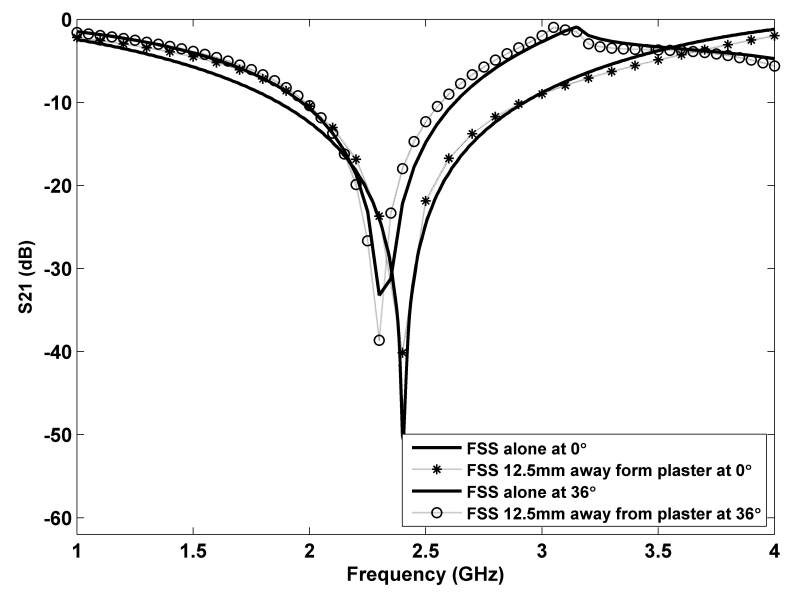

Fig. 15. Comparison of the transmission loss between the case where the square loop is alone and the case where it is placed $12.5 \mathrm{~mm}$ in front of plaster for two angles of incidence.

The first scenario under investigation deals with the effect of deploying the FSS, on the external walls (including floor and ceiling). Results obtained along the estimation root, are shown in Figure 16. The spacing between the receiver locations along the estimation route is $10 \mathrm{~cm}$. Two dipole antennas with $2.15 \mathrm{dBi}$ have been used as transmitting and receiving antenna elements. The transmitting power was set to $10 \mathrm{dBm}$. The obtained results suggest that the field strength inside the building has generally increased whereas the one outside the building has decreased. The power outside the building is 
reduced by roughly $15-20 \mathrm{~dB}$. This effectively means that any interference caused to other wireless systems operating on the same frequency channel outside the external walls should be minimized. The power inside the building is increased by $10-20 \mathrm{~dB}$ depending on the receiver location. It is noted that in areas where radio propagation is dominated by LOS components (e.g. along the corridor), the application of FSS on the external walls had a minimal effect.

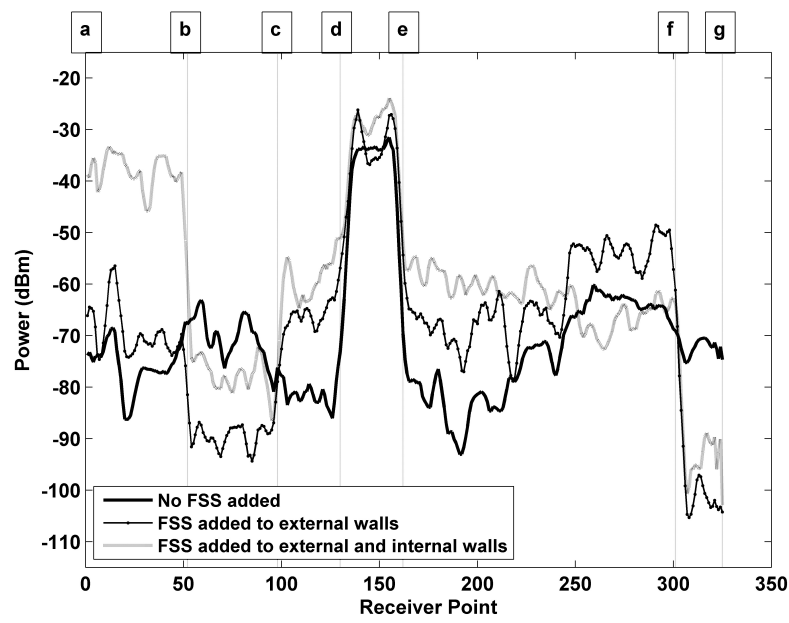

Fig. 16. Comparison of different cases at $2.4 \mathrm{GHz}$. The letters at the top of the figure correspond to the points along the estimation route shown as shown in Figure 14.

Results also suggest that the received field inside the meeting room has not been significantly increased. The next step of this work was to find a way to channel the signal along the corridor and increase the signal strength in this area. To achieve this, FSS were added to the internal walls as well. Results presented in Figure 16 suggest that there is a significant increase of field strength in the meeting room compared to the non-FSS case. It is also noted that the field strength inside office rooms 3,4 and 5 has not decreased since the attenuation suffered by the rays while crossing the internal walls of these rooms is compensated by the stronger multiple reflections along the narrow corridor. However, this kind of compensation does not happen on the received power outside the building, for the case that FSS were added only to the external walls. In this case the ray paths which undergo multiple reflections on the external walls are bigger in length, compared to the paths which undergo multiple reflections along the narrow corridor. This effectively means that these components will suffer higher path loss and they will not contribute significantly to compensate the power reduction due to the transmission through the wall.

Results suggest that in certain scenarios, FSS will act as passive repeaters, channeling the signal to areas of interest while at the same time restricting coverage to other areas.

To highlight the effect of the deployed FSS, tuned at $2.4 \mathrm{GHz}$, a radio transmission at $5.2 \mathrm{GHz}$ was assumed. The transmission and reflection coefficients of the latter frequency were also obtained through the CFDTD method. Figure 17 suggests that the FSS has very little effect on the radio propagation characteristics on this frequency, since the FSS

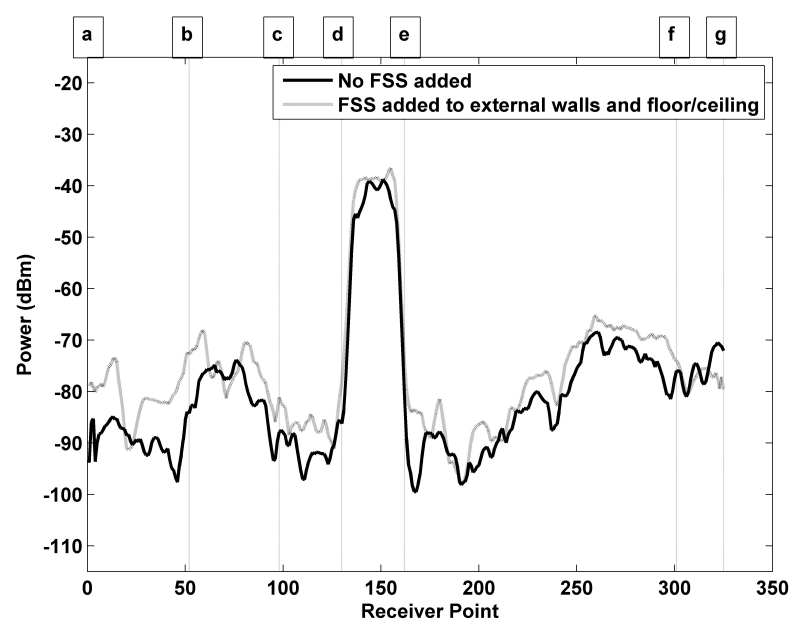

Fig. 17. Comparison of different cases at $5.2 \mathrm{GHz}$

is not tuned at $5.2 \mathrm{GHz}$. This effectively means that carefully designed and deployed FSSs will not have any effect on a system operating on another frequency. An example could be the confinement of WLAN $802.11 \mathrm{~b} / \mathrm{g}$ signals within a building without obstructing the transmission of GSM or 802.11a signals through such a frequency selective environment.

\section{CONCLUSION}

In this paper the basic isolation and passive amplification capabilities of FSS were demonstrated through the use of specially modified Ray Tracing algorithms and anechoic chamber measurements. Simulations and measurements were carried out to verify the angular sensitivity of the reflection and transmission characteristics of the FSS and the effect on their frequency response when the distance between the FSS and the building material is varied. Based on a modified Ray Tracing model, a simple scenario was simulated, highlighting typical isolation and amplification figures that can be obtained. The typical amplification figures were also verified through a small scale experiment in an anechoic chamber. These figures will depend on the specific type and setup of the FSS used. The results suggest that proper FSS deployment can be used in indoor wireless environments in order to increase or restrict coverage and that Ray Tracing techniques can be applied to predict radio propagation in such environments. Proper FSS deployment can assist signal channelling or confine coverage in specific areas.

\section{REFERENCES}

[1] H. Hashemi, The Indoor Radio Propagation Channel, IEEE Proceedings, vol. 81 , no. 7, pp. 943-968, July 1993.

[2] S. Stavrou, S. R. Saunders, Factors influencing outdoor to indoor radio wave propagation, 12th International Conference on Antennas and Propagation, ICAP 2003, pp. 581-585, 31 Mar-3 April 2003.

[3] S. Stavrou, S. R. Saunders, Review of Constitutive Parameters of building material, 12th International Conference on Antennas and Propagation, ICAP 2003, pp. 211-215, 31 Mar-3 April 2003.

[4] M. Yang, S. Stavrou, Investigation of Radio Transmission Losses due to Periodic Building Structures, 11th European Wireless Conference, Nicosia, Cyprus, pp. 737-739, April 2005. 
[5] M. Yang, S. Stavrou, Rigorous Coupled-wave analysis of radio wave propagation through building structures, IEEE Antennas and Wireless Propagation Letters, vol. 3, pp. 204-207, 2005.

[6] N. Monk, H. S. Winbigler, Communication with moving trains in tunnels, IRE Trans. On Veh. Comm., vol. VC-7, pp. 21-28, Dec. 1956.

[7] D. J. R. Martin, Leaky-Feeder Radio Communication: A Historical Review, 34th IEEE Veh. Comm. Conf., vol. 34, pp. 25-30, 21-23 May 1984.

[8] Q. V. Davis, D. J. R. Martin, R. W. Haining, Microwave Radio in Mines and Tunnels, 34th IEEE Veh. Comm. Conf., vol. 1, pp. 375-382, 13 May 1989.

[9] K. J. Bye, Leaky-Feeders for cordless communication in the office, 8th European Conf. on Electronics, E-UROCON 88, pp. 387-390, 1317 June 1988.

[10] Y. P. Zhang, Indoor Radiated-Mode Leaky Feeder Propagation at 2.0 GHz, IEEE Trans. Veh. Tech., vol. 50, no. 2, March 2001.

[11] J.P. Daniel, Mutual coupling between antennas for emission or reception application to passive and active dipoles, IEEE Trans. On Antennas and Propagation, vol. 22, no. 2, pp. 347-349, 1984.

[12] F. A. Chaudhry, M. Raspopoulos, S. Stavrou, Effect of Frequency Selective Surfaces on radio wave propagation in indoor environments, 11th European Wireless Conference 2005, Nicosia, Cyprus, pp. 732-736, April 2005.

[13] R. F. H. Yang, Passive Repeater using double flat reflectors, IRE International Convention Record, vol. 5, Part. 1, pp. 36-41, March 1957.

[14] H. D. Hristov, R. Feick, W. Grote, Improving signal Coverage by the use of through wall passive repeaters, IEEE International Symposium on Antennas and Propagation 2001, vol. 2, pp. 158-161, 17-19 July 2001

[15] Y. Huang, Na Yi, Xu Zhu, Investigation of using passive repeaters for indoor radio improvement, IEEE International Symposium on Antennas and Propagation 2004, vol. 2, pp. 1623-1626, 20-25 June 2004.

[16] C. Colavito, G. D'Auria, Experimental Research on the behavior of Passive Repeaters, IEEE Proceedings, vol. 51, issue 11, pp. 1423-1430, July 1963.

[17] G. H. H. Sung, K. W. Sowerby, A. G. Williamson, The impact of Frequency Selective Surfaces applied to standard wall construction materials, IEEE International Symposium on Antennas and Propagation 2004, vol. 2, pp. 2187-2190, 20-25 June 2004.

[18] M. Raspopoulos, F. A. Chaudhry, S. Stavrou, Radio Propagation in Frequency Selective Buildings, Europ. Trans. Telecoms, Vol. 17, pp. 407413, March 2006.

[19] G. H. H. Sung, et al, A Frequency Selective Wall for Interference Reduction in Wireless Indoor Envrionments, IEEE Antennas and Propagation Magazine, vol. 48, no. 5, pp. 29-37, 2006

[20] G. H. H. Sung, et al, Modeling a Low-cost Frequency Selective Wall for Wireless-Friendly Indoor Environments, Antennas and Wireless Propagation Letters, vol. 5, no. 1, pp. 311-314, 2006.

[21] J. C. Vardaxoglou, Frequency Selective Surfaces - Analysis and Design, Research Studied Press Ltd., England 1997, ISBN: 086380196 X.

[22] B. A. Munk, Frequency Selective Surfaces: Theory and Design, John Willey \& Sons, NY USA. 2000, ISBN 0-471-37047-9.

[23] W. Gregorwich, The design and development of frequency selective surfaces for phased arrays, IEEE Aerospace Conference Proceedings 1999, pp. 471-479, 6-13 March 1999.

[24] R. Mittra, C. H. Chan, T. Cwik, Techniques for analyzing frequency selective surfaces - a review, IEEE Proceedings, vol. 76, issue 12, pp. 1593-1615, Dec. 1988.

[25] W. Yu, R. Mittra, Conformal Finite-Difference Time Domain Maxwell's Equations Solver, Artech House Publishers, November 2003, ISBN: 158053-731-6.

[26] W. Yu, R. Mittra, A conformal FDTD software package modeling antennas and microstrip circuit components, IEEE Antennas and Propagation Magazine, vol. 42, issue 5, pp. 28-39, Oct. 2000.

[27] S. M. A. Hamdy, E. A, Parker, Current Distribution on the Elements of a square loop Frequency Selective Surface, Electronic Letters, vol. 18, No. 14, pp. 624-626, July 1982

[28] R. J. Langley, E. A. Parker, Equivalent Circuit Model for arrays of square loops, Electronic Letters, vol. 18, no. 7, pp. 294-296, 1982.

[29] C. H. H. Sung, K. W. Sowerby, A. G. Williamson, Equivalent circuit Modeling of a Frequency Selective Plasterboard Wall, IEEE Antennas and propagation Int. Symposium, vol. 4A, pp. 400-403, July 2005.

[30] W. Yu, R. Mittra, A look at some challenging problems in computational electromagnetics, IEEE Antennas and Propagation Magazine, vol. 46, issue 5, pp. 18-32, Oct. 2004.

[31] T. K. Wu, Frequency Selective Surface and Grid Array, John Wiley \& Sons Inc., 1995.
[32] P. Callaghan, E. A. Parker, R. J. Langley, Influence of supporting dielectric transmission properties of frequency selective surfaces, IEE Proceedings, vol. 138, no. 5, pp. 448-454, Oct. 1991.

[33] T. Cwik, R. Mittra, The Cascade Connection of Planar Periodic Surfaces and lossy dielectric layers to form an arbitrary periodic Screen, IEEE Trans. Antennas and Propagation, vol. 35, no. 12, pp. 1397-1405, Dec. 1987.

[34] M. Raspopoulos, S. Stavrou, Frequency Selective Surfaces on Building Material - Air gap impact, IET Letters, Vol. 43, Issue 13, pp. 700-702, June 2007.

[35] S. Contu, R. Tascone, Passive Arrays in a stratified dielectric medium scattering matrix formulation, IEEE International Symposium on Antennas and Propagation 1983, vol. 21, pp. 622-625, May 1983.

[36] G. E. Athanasiadou, A. R. Nix, A novel 3-D indoor ray -tracing propagation model: the path generator and evaluation of narrow-band and wide-band predictions, IEEE Transactions on Veh. Tech., vol. 49, issue 4, pp. 1152-1168, July 2000.

[37] Z. Ji, B. H. Li, H. X Wang, H. Y. Chen, T. K. Sarkar, Efficient ray tracing methods for propagation prediction for indoor wireless communications, IEEE Antennas and Propagation Magazine, vol. 43, issue 2, pp. 41-49, 2001.

[38] R. G. Kouyoumjan , P. H. Pathak, A uniform geometrical theory of diffraction for an edge in a perfectly conducting surface, Proceedings of IEEE, vol. 42, pp. 1148-1461,Nov. 1974.

Marios Raspopoulos was born in Nicosia, Cyprus. He received an M.Eng degree in Electronics and Mobile Communications from the University of Surrey, UK in 2003. In 2004 he was awarded a M.Sc. in Communications Networks and Software again from the University of Surrey and then continued his research work into $\mathrm{PhD}$ level at the Centre for Communication Systems Research (CCSR) at the University of Surrey which he received in 2008. His research deals with techniques for improving radio propagation mainly in indoor environments. His work involves deterministic modeling using Ray Tracing, 3D electromagnetic simulations and design of Frequency Selective Surfaces. His other interests include antenna design and UWB Communications, localization techniques and MIMO communications.

Stavros Stavrou was awarded a B.Eng degree in Computer and Communication Engineering from the University of Essex, UK in 1996 and the Ph.D. Degree for the University of Surrey, U.K., in 2001. From 1997 to 2000 he was working as a research fellow in the Centre for Communication Systems Research (CCSR) at the University of Surrey, contributing to a number of international and national research projects. In late 2000 he was appointed to a lecturing position at the University of Surrey, in the area of radio propagation, $\mathrm{RF}$ and antenna design. His main research interests include wireless propagation for terrestrial and satellite systems. 